\title{
Particle Acceleration in Two Converging Shocks*
}

\author{
Xin Wang ${ }^{1,2,3,4,5}$, Joe Giacalone ${ }^{2}$, Yihua Yan $^{3}$, Mingde Ding ${ }^{4}$, Na Wang ${ }^{1,5}$, and Hao Shan ${ }^{1,5}$ \\ ${ }^{1}$ Xinjiang Astronomical Observatory, Chinese Academy of Sciences, Urumqi 830011, China; wangxin@xao.ac.cn \\ ${ }^{2}$ Lunar and Planetary Laboratory, University of Arizona, Tucson AZ 85721, USA \\ ${ }^{3}$ CAS Key Laboratory of Solar Activity, National Astronomical Observatories, Beijing 100012, China \\ ${ }^{4}$ Key Laboratory of Modern Astronomy and Astrophysics (Nanjing University) Ministry of Education, Nanjing 210093, China \\ ${ }^{5}$ Key Laboratory of Radio Astronomy, Chinese Academy of Sciences, Nanjing 210008, China \\ Received 2016 July 18; revised 2017 May 22; accepted 2017 May 23; published 2017 June 15
}

\begin{abstract}
Observations by spacecraft such as ACE, STEREO, and others show that there are proton spectral "breaks" with energy $E_{\mathrm{br}}$ at $1-10 \mathrm{MeV}$ in some large CME-driven shocks. Generally, a single shock with the diffusive acceleration mechanism would not predict the "broken" energy spectrum. The present paper focuses on two converging shocks to identify this energy spectral feature. In this case, the converging shocks comprise one forward CME-driven shock on 2006 December 13 and another backward Earth bow shock. We simulate the detailed particle acceleration processes in the region of the converging shocks using the Monte Carlo method. As a result, we not only obtain an extended energy spectrum with an energy "tail" up to a few $10 \mathrm{MeV}$ higher than that in previous single shock model, but also we find an energy spectral "break" occurring on $\sim 5.5 \mathrm{MeV}$. The predicted energy spectral shape is consistent with observations from multiple spacecraft. The spectral "break," then, in this case is caused by the interaction between the CME shock and Earth's bow shock, and otherwise would not be present if Earth were not in the path of the CME.
\end{abstract}

Key words: acceleration of particles - methods: numerical - scattering - shock waves -

Sun: coronal mass ejections (CMEs) - turbulence

\section{Introduction}

In an interplanetary (IP) shock, a proton energy spectral "break" (separating two power laws) can be measured by in situ instruments on multiple spacecraft. There are six events, occurring on 1997 November 6, 2001 February 15, 2005 January 20, 2005 September 7, 2006 December 5, and 2006 December 13, with hard energy spectra. These six large events all show their spectral "breaks" in the energy range of 1-10 MeV. In addition, another six large events from solar cycle 23 occurred on 2000 July 14, 2000 November 8, 2001 September 24, 2001 November 04, 2001 November 22, and 2003 October 28. These events all show spectral "breaks" at about $\sim 50 \mathrm{MeV}$ (Mewaldt et al. 2008). In galactic cosmic-ray (CR) sources, supernova remnants (SNRs) IC 443 and W44 exhibit gamma-ray bumps at energy $\sim 100 \mathrm{MeV}$, from which their parent proton energy spectral "breaks" can be deduced. The "broken" spectra are considered to arise from the interaction between the SNR shocks and molecular clouds (Ackermann et al. 2013). In the present paper, we discuss the 2006 December 13 shock event in interplanetary space, in which proton fluxes show an energy spectral "break" at $\sim 3.5 \mathrm{MeV}$, as measured by the spacecraft ACE, STEREO, and SAMPEX.

A number of in situ observations exhibit the CRs' proton spectral "breaks" associated with the interaction between the source and its environment. Desai et al. (2016) suggest that double power-law solar energetic particle (SEP) spectra occur due to diffusive acceleration by near-Sun CME shocks rather than due to scattering in interplanetary turbulence. Here, we suggest that the energy "break" is associated with the shockinteraction regions. So, we try to perform a reliable prediction

\footnotetext{
* This work is supported by the Xinjiang Natural Science Foundation No. 2014211 A069.
}

of "breaks" using numerical methods. Normally, numerical simulation usually builds a simple diffusive shock acceleration (DSA) model with a small diffusive region ahead of the shock. But the energy spectral "break" is usually associated with a large diffusive size, so the energy spectral "break" would hardly be involved in the simulated result. Technically, the hybrid and particle-in-cell (PIC) simulation methods solve the equation explicitly for particle motions in an electromagnetic plasma (Giacalone et al. 1993; Guo \& Giacalone 2013; Yang et al. 2015). The PIC and hybrid methods can directly model not only the particle acceleration process but the shock formation process as well. Both of them have the great advantage in that they determine the self-generated magnetic turbulence self-consistently. However, the extensive energy spectra for resolving issues such as the energy spectral "break" are not easy to obtain. Nevertheless, the Monte Carlo method (Ellison \& Eichler 1984; Wang \& Yan 2011; Wang et al. 2013) solves the Boltzmann equation using the collective scattering technique, replacing the explicit calculation of the electromagnetic field in the shock region. The scattering mean free path is assumed to be a function of the particle rigidity. Theoretically, this treatment allows the individual ions and large diffusive region to be followed for a long time until a sufficient high-energy "tail" arises. Actually, the acceleration efficiency and the maximum particle energy are dependent on the size of the precursor region, which is parameterized by the size of the free escape boundary (FEB) in the Monte Carlo single shock model. Using a limited computation, Ellison et al. (1990) presented an ion spectrum with a maximum particle energy less than $1 \mathrm{MeV}$ by applying a fixed FEB size ahead of the bow shock. Knerr et al. (1996) and Wang \& Yan (2012) improved the simulated result for the maximum particle energy up to $\sim 4 \mathrm{MeV}$ using a moving FEB ahead of the shock. The simulations of Wang et al. (2016) revealed a maximum energy 
of about $5.5 \mathrm{MeV}$ in the isolated shock model, which is not high enough to investigate the spectral "breaks" seen in the CME events. It is still difficult to use an isolated shock model to solve this energy spectral "break."

Since SEP events are important both dynamically and diagnostically, it is essential that we understand their acceleration, transport, radiative emissions, and interaction with nearby environments. There are several possible models for the energy spectral "break." The first model proposes that the "break" is determined by different chemical compositions of SEPs. A heavy nucleus with a charge number of $Z$ has maximum energies $Z$ times that of a proton with the same Larmor radius. This leads to heavy ions having more difficulty escaping from the acceleration site than protons, and a "break" is formed at a certain energy range (Prouza \& Smida 2003). The second model suggests that the "break" is probably associated with the leakage mechanism. It means that CRs can drive Alfvén waves efficiently to build a transport barrier. This barrier can strongly reduce the leakage of particles and lead to an occurrence of the energy spectral "break" (Malkov et al. 2013). The third model proposes that the "break" would be formed in the site of the drifting shock that interacts with their surrounding media. For example, if an SNR blast wave approaches the termination shock of the stellar wind and a system of colliding shock flows forms, the confinement of accelerated particles will deteriorate and would lead to an energy spectral "break" (Bykov et al. 2013a, 2013b). Also, a multiple-shock model assumes that the medium is highly turbulent and that an ensemble of shocks are propagating through it, which may modify the resulting spectral index to form the spectral shape such as "breaks" (Melrose \& Pope 1993; Schneider 1993; Ferrand \& Marcowith 2010). Here, we propose that the converging shock interaction between the CME-driving shock and Earth's bow shock would probably produce the energy spectral "break."

So, how to extend the shock's energy spectral "tail" to higher energies is the key to solving the energy spectral "break" by numerical simulation. In order to confirm the IP shock's energy spectral "break," we should follow particles that produce an energy spectrum with an extensive energy range beyond the "broken" energy. In our previous works focusing on isolated shocks, our simulated spectrum did not extend to high-enough energies to predict the energy spectral "break." In our present work, we focus on a double-shock interaction (i.e., the CMEdriven shock interacting with Earth's bow shock), which increases the maximum energy. If a sufficiently extensive energy spectrum is available, we will have an opportunity to investigate the energy spectral slope as long as there exists a "broken" energy spectrum, as described in the observed energy spectrum of 2006 December 13. In the converging shock model, there are two reasons that make this investigation is possible. First, the converging shocks can inject more accelerated particles into the first-order Fermi acceleration mechanism and produce a more extensive energy spectrum than the isolated shock model. Second, the double shocks can provide a fraction of the accelerated particles with more frequent shock encounters to gain more energy and to produce a high-energy "tail." With the compression of the shock precursor region, however, fewer particles will gain more energy from the double-shock crossings and thus lead to a sharply steep energy spectrum, which would differ from the smooth energy spectral shape in an isolated shock model.
In the present paper, we perform simulations to further investigate the energy spectrum in two converging shocks using the Monte Carlo method. In Section 2, we introduce the converging shocks model. In Section 3, the simulated results and analyses are presented. In Section 4, we give the summary and conclusions.

\section{Model}

The Solar and Heliospheric Observatory (SOHO) coronagraphs of the halo CME event on 2006 December 13 show that the CME moved with a speed of $1774 \mathrm{~km} \mathrm{~s}^{-1}$. The fluxes of the protons in this SEP event are measured by the ACE, STEREO, and $S A M P E X$ spacecraft in different energy ranges from 0.1 to $500 \mathrm{MeV}$. The energy spectral "break" appears at energy $\sim 3.5 \mathrm{MeV}$. Since most of the individual spacecraft cannot measure an entire energy spectrum for an SEP event because of their limited field of view, we present a simulation of the double-shock interaction between the CME shock and the Earth's bow shock at the site of the first Lagrangian point (L1). We expect to obtain an extended energy spectrum, which can cover the energy range from $\sim 0.1 \mathrm{MeV}$ to a few decades of $\mathrm{MeV}$. We also hope to investigate this extended energy spectrum involving a possible energy spectral "break" between 1 and $10 \mathrm{MeV}$.

Figure 1 shows a schematic diagram of the converging shock model. The left reflective wall represents a CME and produces a shock wave with a shock velocity of $V_{\text {sh2 }}$ propagating from the left boundary to the center of the box. The bulk flow speed in the upstream region of the CME-driven shock is $U 0_{2}$. The right reflective wall represents Earth and produces a bow shock with a shock velocity of $V_{\text {sh1 }}$ propagating from the right boundary to the center of the box. The bulk flow speed upstream of the Earth's bow shock is $U 0_{1}$. The relative speed between the bulk flows of the upstream of the two shocks is equal to $U 0=\left|U 0_{1}\right|+\left|U 0_{2}\right|$ in the laboratory reference frame. Nevertheless, the relative evolving velocity between the two shock fronts is equal to $V_{\mathrm{sh}}=\left|V_{\mathrm{sh} 1}\right|+\left|V_{\mathrm{sh} 2}\right|$ in the laboratory reference frame. The bulk flow speeds of both downstream regions have the same velocity of zero in the laboratory reference frame. This model describes the double shocks' interaction that occurred on 2006 December 13 at the first Lagrangian point (L1) near Earth. We consider a plane-parallel shock where the supersonic flow moves from the Sun to the Earth (in the rest frame) along the $x$-axis direction. The shock was observed by the WIND, SOHO, and $A C E$ spacecraft near Earth at the location of the first Lagrangian point, $\mathrm{L} 1 \sim 1.5$ million $\mathrm{km}\left(\sim 250 R_{e}\right.$, where $R_{e}$ is the radius of the Earth) upstream of the Earth on 2006 December 13. With the CME-driven shock propagating from the Sun along the $x$-axis to the Earth, its shock front was encountered by the WIND, SOHO, and ACE spacecraft located in $X_{\mathrm{GSE}}$ (Geocentric Solar Ecliptic system) between $250 R_{e}$ and $180 R_{e}$ upstream from the Earth. The distances of all these spacecraft from the Sun -Earth line were within $50 R_{e}$ along the $Y_{\mathrm{GSE}}$ and $Z_{\mathrm{GSE}}$ directions (Liu et al. 2008). According to the WIND magnetic cloud list (https://wind.nasa.gov $/ \mathrm{mfi} / \mathrm{mag}$ _cloud_CA1.html), the 2006 December 13 shock event originated from the western hemisphere of the Sun with an interplanetary "magnetic cloud" axis orientation with a latitude of $\theta=27^{\circ}$ and a longitude of $\phi=85^{\circ}$ in GSE coordinates (von Rosenvinge et al. 2009). From the perspective of the WIND, SOHO, ACE, and STEREO spacecraft, the observed CME-driven shock is a quasi-parallel diffusive shock, so their observations provided an example of a 
The Schematic Diagram of the Simulation Box in Double Shocks Model

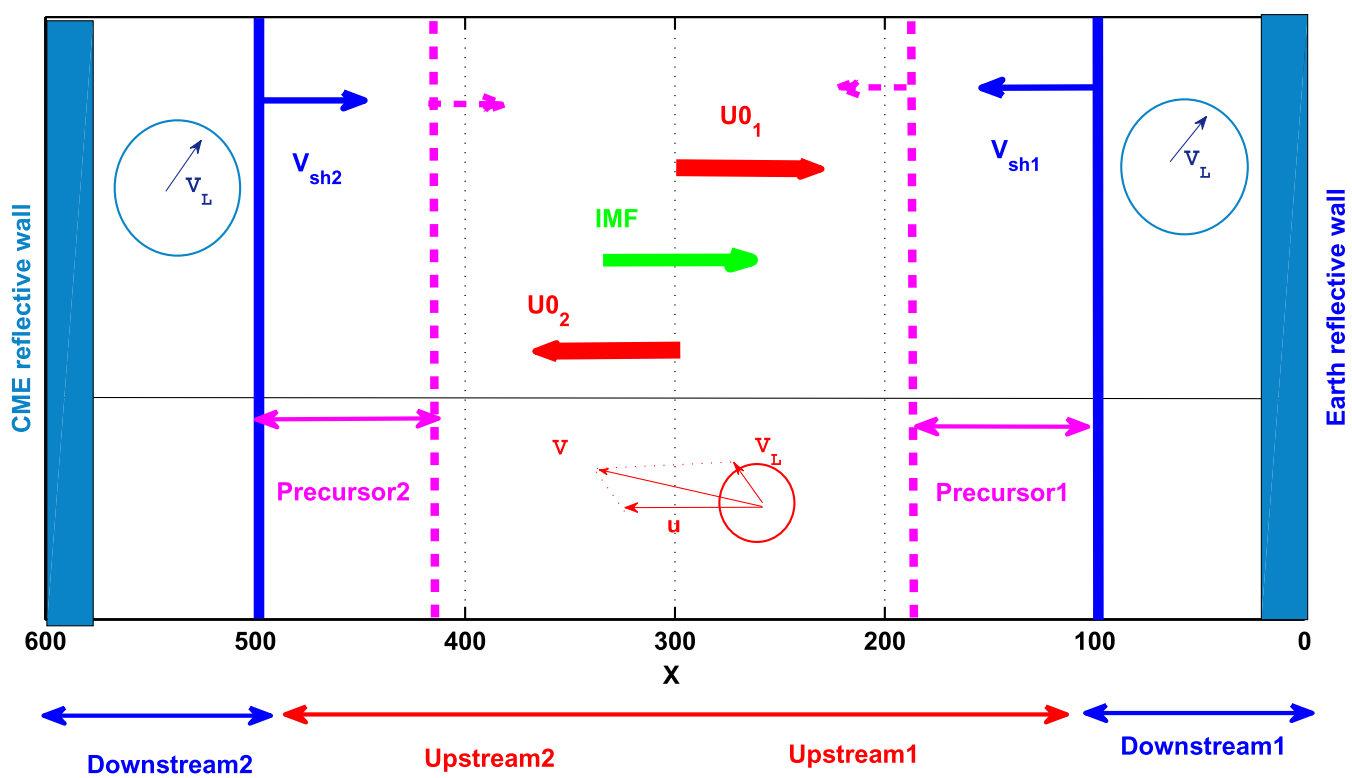

Figure 1. Schematic diagram of the simulation box. The left reflective wall represents a CME and produces shock No. 2, propagating from the left boundary to the center of the simulation box with $V_{\text {sh2. }}$. The right reflective wall represents the Earth and produces shock No.1, evolving from the right boundary to the center of the simulation box with $V_{\mathrm{sh} 1}$. Particles diffuse the turbulence of the amplified magnetic field in the coupled precursor regions between two shock fronts when they gradually evolve forward to the center of the simulation box.

quasi-parallel shock interacting with the Earth's bow shock. In this case, we can apply our dynamic Monte Carlo code to understand the particle acceleration and energy spectral "break" issues based on double-shock interactions. In this model, the CME shock gives a shock normal with an elevation angle of $\theta=27^{\circ}$ to the upstream magnetic field, so the shock may be quasi-parallel and the shock can be taken as an oblique shock with a factor of $\cos \theta\left(\theta=27^{\circ}\right)$. So, we take the relative total speed between the two bulk flows with a modified value of $\sim 1600 \mathrm{~km} \mathrm{~s}^{-1}$ aligned with the interplanetary magnetic field. For simplicity, both shocks are driven by the same speed of the upstream bulk flow with $\left|U 0_{1}\right|=\left|U 0_{2}\right| \sim 800 \mathrm{~km} \mathrm{~s}^{-1}$, but with an opposite direction in the laboratory frame. Also, both shock fronts propagate with opposite velocities of $V_{\mathrm{sh} 1}$ and $V_{\mathrm{sh} 2}$ from each boundary to the center of the box, respectively.

In this Monte Carlo method, we use an initial number density of particles $n_{0}$ obeying a Maxwellian distribution with a local velocity $V_{L}$ in the upstream region. The initial particles, with their bulk flow speeds of upstream and local velocities $\left(V=U+V_{L}\right)$, move to the corresponding reflective wall at each side of the simulation box (here, $U$ represents $U 0_{1}$ or $U 0_{2}$ ). Bulk flows are reflected by each wall and form two downstream regions with high densities of downstream flow on each side of the simulation box. When both downstream flow densities reach their stable states, the two shock fronts then smoothly evolve forward to the center of the box with evolutional velocities $V_{\mathrm{sh} 1}$ and $V_{\mathrm{sh} 2}$, respectively. Also, when both shock fronts propagate closer together, the two precursor regions ahead of their shocks have interactions that gradually approach the center of the simulation box. The interactions of the two precursor regions in the converging shocks lead to an amplified magnetic field between the two shocks. The particles in the two converging shocks gain more energy from this amplified magnetic turbulence than those in a single shock model. Thus, the amplified magnetic field could contribute to the extensive energy spectrum. However, with the shortening of the precursor regions, fewer particles can obtain more energy from double shocks to contribute to the steeply high energy spectral "tail." Therefore, the energy spectral "break" would form at a certain energy range between 1 and $10 \mathrm{MeV}$.

The essential property of the shock lies in its capability to slow down the upstream flow over the short distance $\Delta_{\text {sh }}$ (i.e., the width of the shock transition region). A substantial amount of upstream flow energy must therefore be converted into the heating and compression of the bulk plasma, and the increasing pressure within the $\Delta_{\mathrm{sh}}$ region. Then, the shock produces a downstream region of high temperature but low ram pressure and separates it from the low-thermal high-ram pressure upstream region. The shock is in a non-equilibrium thermal state due to a transparent boundary between the two regions of different pressures. Therefore, in order to be maintained for long periods, the shock should continuously evolve at the expense of energy and momentum density inflow from the shock-upstream region. In addition, when dealing with the acceleration of particles at shocks, the shock presents as a discontinuity between two independent regions of different bulk flow parameters, which are filled with scattering centers for the particles. The turbulent fluctuations appear as scattering centers frozen into the upstream and downstream bulk flows. The Weibel-generated downstream magnetic fields form largeamplitude vortices which could be convected by the downstream flow to large distances from the shock and possibly contribute to an extended strong field region. If CRs are included, the non-resonant models can generate upstream magnetic turbulence at short and long wavelengths in nearly parallel magnetic field shocks (Bykov \& Treumann 2011). The scattering process is usually modeled by some dependence of the mean free path of the particles on the momentum in most particle simulation cases. Thus, in order for the acceleration to be efficient, a large number of shock crossings and reflections back and forth is required. Shock particle acceleration therefore depends on the scattering process, which is clearly stochastic, 
depending on the presence of scattering centers upstream and downstream and on the random changes in the scattering angle. It assumes that the scattering is elastic and isotropic for energy conservation (Treumann \& Jaroschek 2008). This mechanism always works until the gyroradius of the accelerated particle becomes so large that it exceeds the size of the system or until the energy of the particle becomes so large that the backscattering of the particle becomes ineffective. In order to experience a first scattering, the particle must initially already possess a gyroradius much larger than the entire width of the shock transition region. Only when this condition is satisfied will the shock behave like a slightly thin discontinuity separating two regions of very different velocities such that the particle, when crossing back and forth over the shock, becomes aware of the bulk difference in speed and gains energy. In fact, in order to undergo Fermi shock acceleration, a particle must be pre-accelerated or pre-heated until its gyroradius becomes sufficiently large (Treumann 2009). In the regular background solar wind, the background magnetic field turbulence is very weak without the Fermi acceleration for ions. When the high-speed background flow is reflected by the obstacles, the downstream flow turbulence dominated by the Weibel model can form enhanced turbulence fluctuations near the shock front. In this simulation, we use two reflective walls at both sides of the simulation box to produce the CME shock and the Earth's bow shock to slow down the high speed of the upstream inflow at the middle of the simulation box. The double compressional high-density downstream regions at both sides of the simulation box form double boundaries as the shock fronts separate to the middle upstream inflow. With the high-density downstream bulk flows expanding their sizes away from the two reflective walls at the sides of the simulation box, both shock fronts continue to slow down the high-speed upstream inflows at the center of the simulation box.

Our Monte Carlo model uses a scattering law which leads to the ordinary diffusion of particles. We assume that the particles scatter elastically off the background scattering centers with their scattering angles obeying an isotropic distribution in their local frame. In this scattering scenario, the assumption of elastic scattering requires the scattering centers to be frozen into the background fluid (Ellsion \& Double 2004); simultaneously, the assumption of a constant scattering time (i.e., collision time) for all particles means that the particle's mean free path is proportional to its local velocity in the local frame. For simplicity, we take its formula to be

$$
\lambda=V_{L} \cdot \tau,
$$

where $\tau$ is the average scattering time. The scattering time for an individual particle is the duration of the change from the old momentum to the new momentum after collision with the background field at random variations of the pitch angle and azimuth angle. We follow the large number of particle movements in three substeps: (i) Particle movement. Particles move with their velocities along the one-dimensional $x$-axis, which is parallel to the magnetic field direction. (ii) Momentum collection. The summations of the particle masses and velocities are calculated at the center of each computational grid for subsequent use. (iii) Applying isotropic scattering law. A certain fraction of the particles are chosen to scatter the background scattering centers with their random scattering angles. The average number of scattering events occurring in
Table 1

The Simulation Parameters

\begin{tabular}{llc}
\hline \hline Physical Parameters & Dimensionless Values & Scaled Values \\
\hline Upstream bulk speed 1 & $U 0_{1}=-0.6$ & $-800 \mathrm{~km} \mathrm{~s}^{-1}$ \\
Upstream bulk speed 2 & $U 0_{2}=0.6$ & $800 \mathrm{~km} \mathrm{~s}^{-1}$ \\
Initial thermal velocity & $v_{L}=0.02$ & $26.9 \mathrm{~km} \mathrm{~s}^{-1}$ \\
Scattering time & $\tau_{0}=0.833$ & $0.13 \mathrm{~s}$ \\
Box size & $X_{\max }=600$ & $2000 R_{e}$ \\
Total time & $t_{\max }=2400$ & $630 \mathrm{~min}$ \\
Time step size & $d t=1 / 15$ & $0.01 \mathrm{~s}$ \\
Number of zones & $m_{x}=1200$ & $\ldots$ \\
Initial particles per cell & $n_{0}=360$ & $\ldots$ \\
\hline
\end{tabular}

Note. $R_{e}$ is the Earth's radius. The scale factors for distance, velocity, and time are $X_{\text {scale }}=2000 R_{e} / 600, U_{\text {scale }}=800 \mathrm{~km} \mathrm{~s}^{-1} / 0.6$, and $t_{\text {scale }}=X_{\text {scale }} / U_{\text {scale }}$. The dimensionless values and the scaled values can be transformed by the scaled factors to each other.

an increment of time $d t$ depends on the average scattering time $\tau$, and the scattering rate is given by

$$
R_{s}=d t / \tau
$$

where $R_{s}$ is the probability of the scattering events occurring in an increment of time. The candidates with their local velocities and scattering angles scatter off the grid-based scattering centers. These individual particles do not change their routes until they are selected to scatter once again. For an individual proton, the grid-based scattering center can be seen as the sum of individual momenta. So, these scattering processes can be taken to be elastic collisions. In an increment of time, once all of the candidates complete these elastic collisions, the momentum of the grid-based scattering center is changed. The total simulation temporally evolves forward by repeating the time step sequence. To calculate the scattering processes accurately and produce an exponential mean free path distribution, the time step should be less than the scattering time (i.e., $d t<\tau$ ). In these diffusive processes, the particles in the upstream region transfer their kinetic energy into their random thermal energy in the downstream region. Then, a few random thermal particles can diffuse back from the downstream region to the upstream region over multiple scattering cycles on shock fronts to gain more energy and become superthermal particles.

All the simulated parameters are listed in Table 1. According to the observation, we adjust the observed parameters to apply to the simulation. We present the scaled values of the parameters as follows. The upstream bulk flow speed toward the Earth is $-800 \mathrm{~km} \mathrm{~s}^{-1}$, and the upstream bulk flow speed toward the CME is $800 \mathrm{~km} \mathrm{~s}^{-1}$. The initial thermal velocity is $v_{L}=26.9 \mathrm{~km} \mathrm{~s}^{-1}$. The scattering time is $\tau_{0}=0.13 \mathrm{~s}$. The box size is set to $2000 R_{e}$ (where $R_{e}$ is the Earth radius). The total time of the simulation is 630 minutes, and the time step is set to be $0.01 \mathrm{~s}$.

The above scaled values of the parameters correspond to the following dimensionless parameters: upstream bulk speeds $U 0_{1}=-0.6$ and $U 0_{2}=0.6$, initial local thermal velocity $v_{L}=0.02$, the constant of the scattering time $\tau_{0}=0.833$, the total size of the box $X_{\max }=600$, the total simulation time $t_{\max }=2400$, and the time step $d t=1 / 15$. These above dimensionless values can be scaled by the distance $(X)$, time $(t)$, 
and velocity $(U)$ scaling factors: $X_{\text {scale }}=2000 R_{e} / 600$, $U_{\text {scale }}=800 \mathrm{~km} \mathrm{~s}^{-1} / 0.6$, and $t_{\text {scale }}=X_{\text {scale }} / U_{\text {scale }}$, respectively.

In addition, we give the simulation box grids of $m_{x}=1200$, and the initial density of particles in each grid is $n_{0}=360$. So, the total number of particles in the simulation box at the end of the simulation is statistically going to be more than $1,000,000$ particles.

\section{Results}

\subsection{Shock Evolution}

In Figure 2, the left panel shows a group of bulk flow velocity snapshots with the grids at the simulation time of $t=720,1200$, and 1680 . The right panel shows the density snapshots with the grids at the simulation time of $t=960$, 1440, and 1920. In the left panel, the bulk flows with initial velocities $U 0_{1}$ and $U 0_{2}$ in their upstream regions evolve into their downstream regions with bulk flow speeds of zero, respectively. With the time increasing, the two downstream regions expand from the two sides to the center of the simulation box, respectively. The two shock fronts also move away from the two boundaries to the center of the box with opposite shock velocities $V_{\mathrm{sh} 1}$ and $V_{\mathrm{sh} 2}$, respectively. Ahead of each shock, the velocity profiles in their respective upstream regions exhibit gradual slopes, which represent shock precursor regions caused by the pressure of the energetic particles accelerated by the shocks. When two shock fronts approach each other, the two precursors overlap, leading to irregularities in the magnetic field. The interaction of the two precursors may lead to changes in the energy spectrum of the energetic particles. In the right panel, the bulk flow density with an initial number density $n_{0}$ in each grid evolves into the downstream regions with several times the initial density, respectively. Considering this simulation containing two shock regions, doubling the simulation box size and the total particle number of a single shock would lead to expensive computations. We technically use a value of the particle density in the pre-inflow (i.e., the new particles preparing to enter the box as the new upstream bulk flows at the center of the box moving toward both shock downstream regions) that decreases with the time; we note that the densities of the two downstream regions exhibit a slight decrease as time increases. To speed up the computation, we specifically use an adaptive inflow density with a density reduction of $\Delta n=16$ in each time duration (here, the total simulation time $t_{\max }=2400$ is divided into 10 time durations along the time axis represented by $Q=1,2$, $3, \ldots, 10$ in sequence). We have found that the adaptive inflow density did not significantly alter the simulated energy spectrum. As shown in the bottom-right panel of Figure 2, the density profiles can also give rise to an interaction of the overlapping precursor regions at the simulation time of $t=1920$.

Figure 3 shows a group of bulk flow velocity profiles at durations of $Q=1,3,5$, and 7 and density profiles at durations of $Q=1,3,5$, and 7. The red solid line in each upstream region represents the precursor position. The left panel shows a series of bulk flow velocity profiles in terms of position with time. In the laboratory reference frame, the bulk flow velocities in the two downstream regions are equal to zero, and the velocities of the bulk flow in the two upstream regions show opposite movements in the middle of the simulation box. The bottom-left mesh plot, indicated by the index of the time sequence $Q=7$, shows that the two precursor regions overlap as the time increases. The right panel shows a series of density profiles of the position with the time. Three mesh plots indicate that the higher density of the downstream region on each side shortens the upstream region, which has a low density, as the time moves from $Q=1,3,5$, to 7 , respectively. The bottomright mesh plot indicated by the index of the time sequence $Q=7$ shows the two precursor regions mixed together, with the upstream regions gradually disappearing. Here, the overlapping precursor regions may play two important roles for the energetic particles. (i) Energetic particles gain more energy from the amplified magnetic field turbulence excited by the interaction of the double shocks and produce a higher maximum energy compared to one shock. (ii) With the shortening precursor region, fewer and fewer particles can cross the double shocks and accelerate, and thus naturally produce a softer spectrum at the higher energy range. We suggest that the compressed precursor region would be responsible for the occurrence of the energy spectral "break."

\subsection{Particle Acceleration}

Figure 4 shows a group of plots showing the trajectories of individual particles at different times $Q=5,6,7,8,9$, and 10 . A few particles extracted from the simulation box are represented by the blue curves in each plot with the corresponding index of the time sequence $Q$. The shaded areas represented by the two triangles in each plot indicate that two shock fronts move forward to the center of the simulation box from both sides of the simulation box as the time increases, respectively. Some of the curves show particle acceleration by crossing either one of the two shock fronts back and forth with energy gains. Another curve at the bottom of each plot shows particles without acceleration in the downstream regions. The maximum particle velocities $V_{\max }$, with values of 31.3898 , $33.5277,35.4340,37.3926,37.5091$, and 36.4455, are calculated at the durations of $Q=5,6,7,8,9$, and 10, respectively. These maximum velocities show that the particles keep accelerating up to a saturation value at a certain time. The maximum energy at $Q=10$, as indicated in the bottom-right plot of the panel, exhibits a value less than the saturation value in the bottom-left plot of the panel. It can prove the negative effect of the shortening precursor size on the accelerated particles as the time proceeds from $Q=8,9$ to 10 . Although the merged precursor region could lead to an amplified magnetic field that will produce a more energetic particle population than that produced in a single shock model, it could prevent the CME shock from following a regular single powerlaw spectrum. Actually, the compressed precursor region could lead to a steep slope at higher energies. Because fewer particles randomly walk across the two converging shocks, the resulting energy spectrum would have a spectral "break"-the total energy spectrum would possibly "break" down at a certain energy range of a few $\mathrm{MeV}$.

\subsection{Energy Spectra}

Figure 5 shows the plot of the simulated proton energy spectrum and the energy spectrum for the event we have studied that was observed by $A C E$ and STEREO. For convenience, the simulated proton flux is scaled in the same integrating duration as the observed flux that occurred in the period from 2006 December 13, 02:00 to December 14, 22:00, 

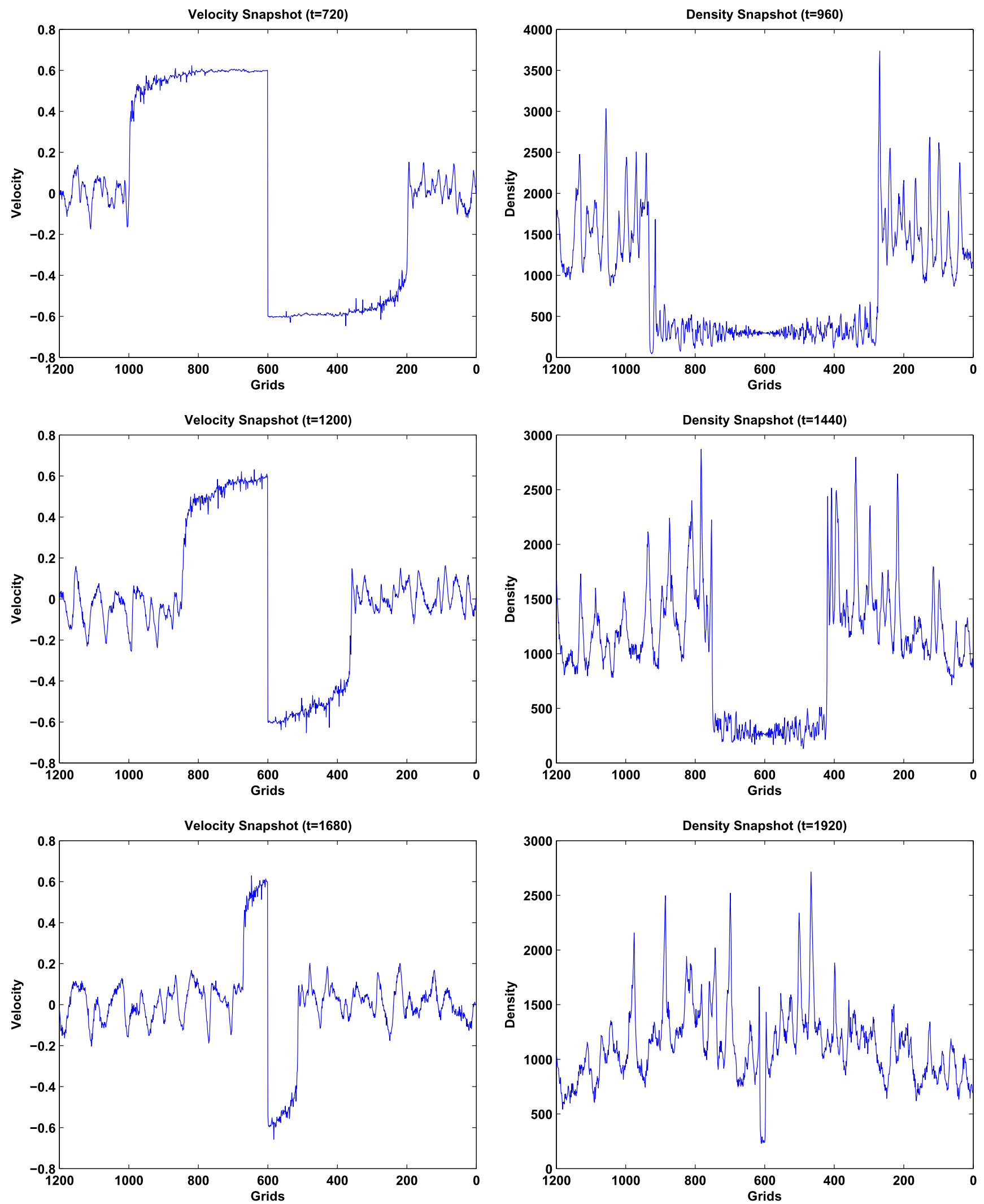

Figure 2. The three left subplots represent snapshots of the bulk flow velocity with the grids at the simulation time $t=720,1200$, and 1680 . The three right subplots represent snapshots of the density of the bulk flow with the grids at the simulation time $t=960,1440$, and 1920.

which is equivalent to $1.584 \times 10^{5} \mathrm{~s}$. The plot shows a comparison of the simulated energy spectrum and that observed. Each shows a spectral feature in the kinetic energy range from 0.1 to $20 \mathrm{MeV}$. The blue curve represents the observed energy spectrum, which shows the double power-law energy spectrum with a "break." The lower energy spectrum shows an energy spectral shape with a slope of $E^{-1.07}$; the higher energy spectrum shows a softer energy spectral shape 

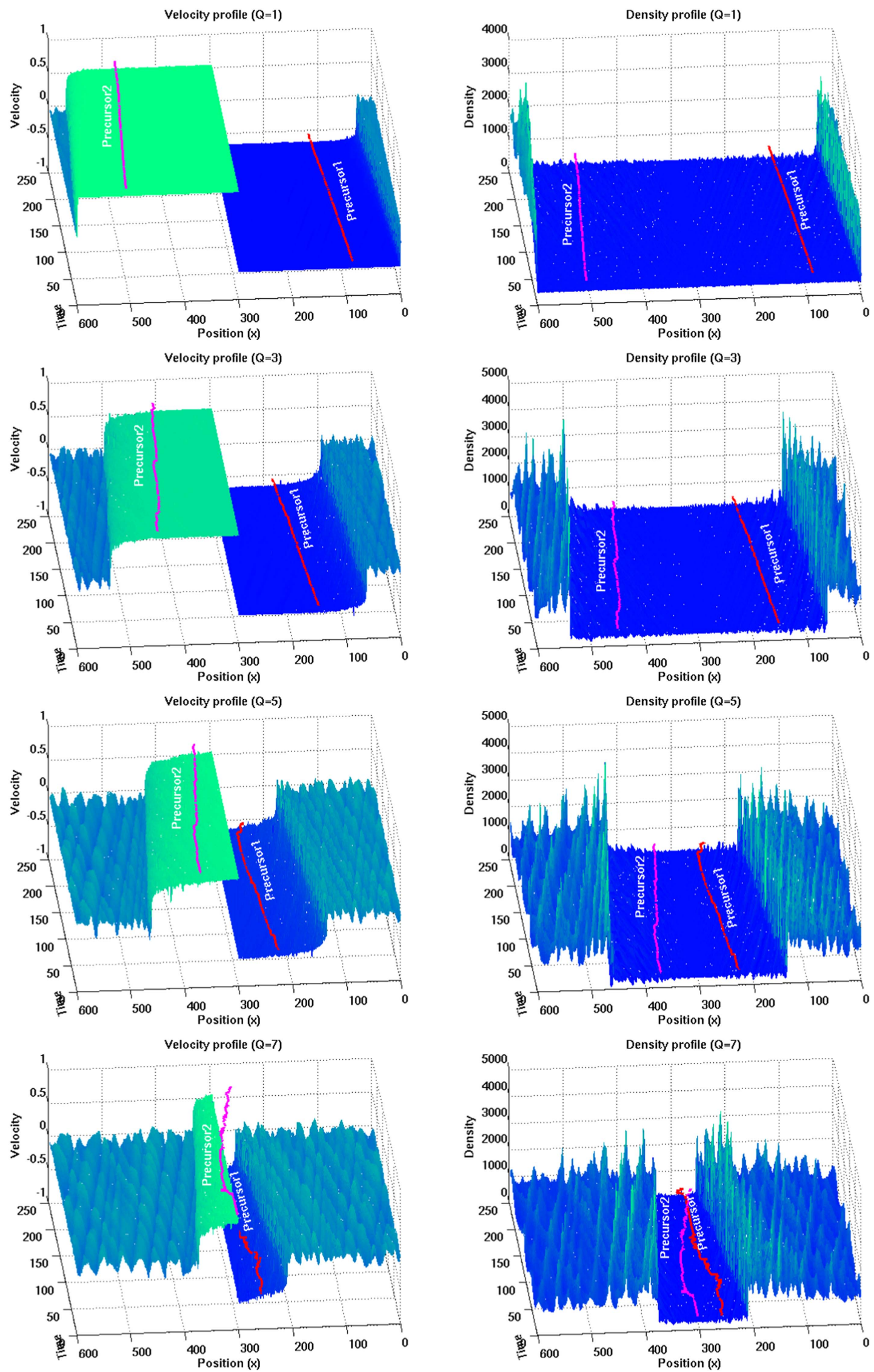

Figure 3. The left panel represents the profiles of the bulk flow velocity at durations of simulation time $Q=1,3,5,7$. The right panel represents the profiles of the bulk flow density at durations of simulation time $Q=1,3,5,7$. The red solid line in each upstream region represents the precursor region boundary, respectively. 

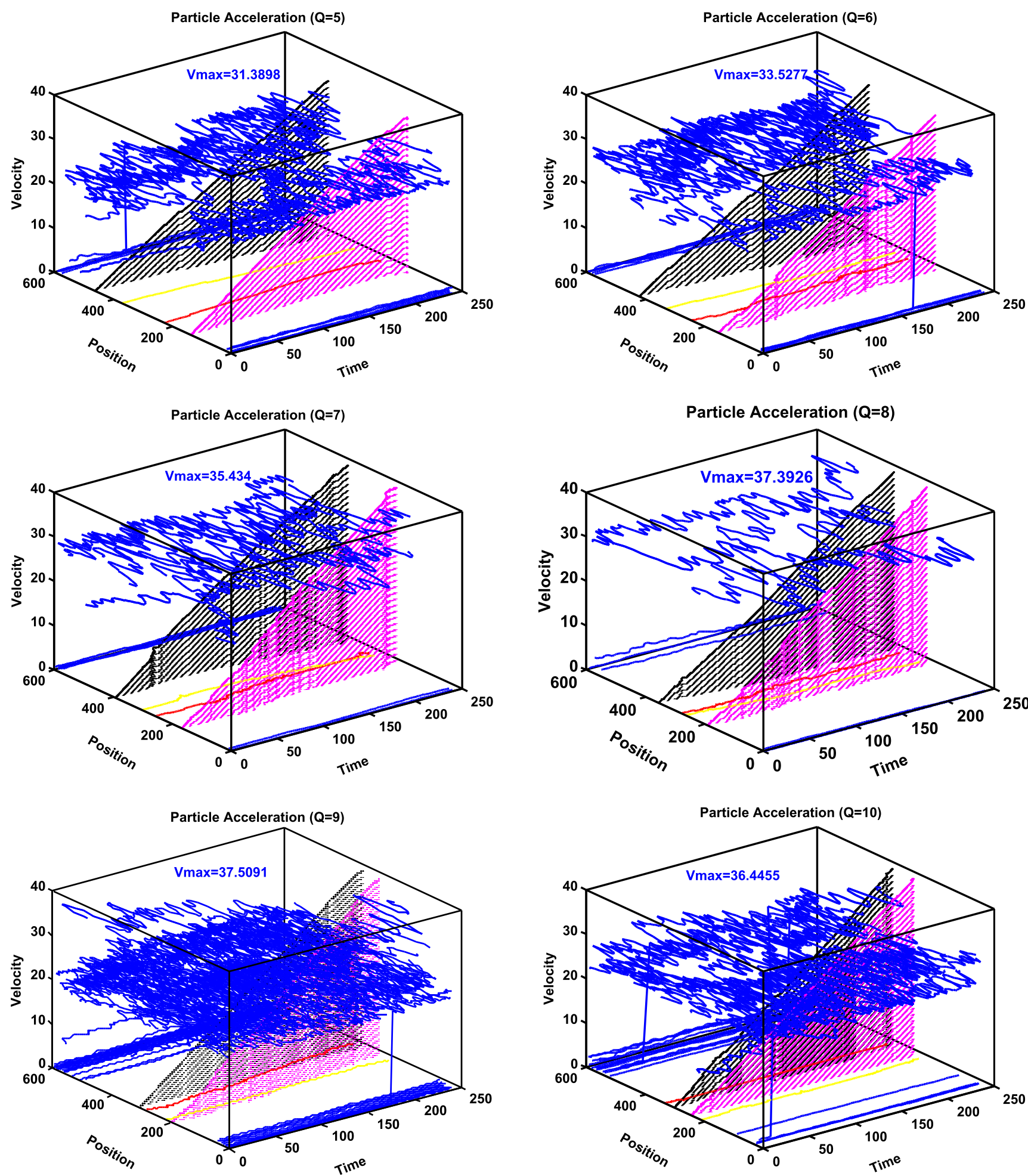

Figure 4. This group of plots represent particle acceleration in double shocks at the durations of simulation time $Q=5,6,7,8,9$, and 10 . The curves in each plot represent particle trajectories. These trajectories show the evolutions of the particles' local velocities vs. their positions and time.

with a slope of $E^{-2.45}$. The spectral "break" energy occurs at $\sim 3.5 \mathrm{MeV}$. The red curve represents the simulated energy spectrum, which also shows a spectral "break." The black vertical dashed line indicates the position of the energy spectral "break." The cyan line to the left of the vertical dashed line represents the lower energy spectrum with an energy slope of $E^{-1.17 \pm 0.11}$, which exhibits a difference in the fluxes compared with the observed energy spectrum at the lower energy range.
To the right of the vertical dashed line, the green and pink lines fit the higher energy spectrum with two different energy spectral slopes of $E^{-2.55 \pm 0.10}$ and $E^{-2.48 \pm 0.12}$, respectively. At the higher energy range, the pink line with a slope of $E^{-2.48 \pm 0.12}$ would be more similar to the observed energy spectrum with a slope of $E^{-2.45}$, but it deviates more toward the "break" point at the vertical dashed line. In comparison, the green line with a slope of $E^{-2.55 \pm 0.10}$ would be more fit for 


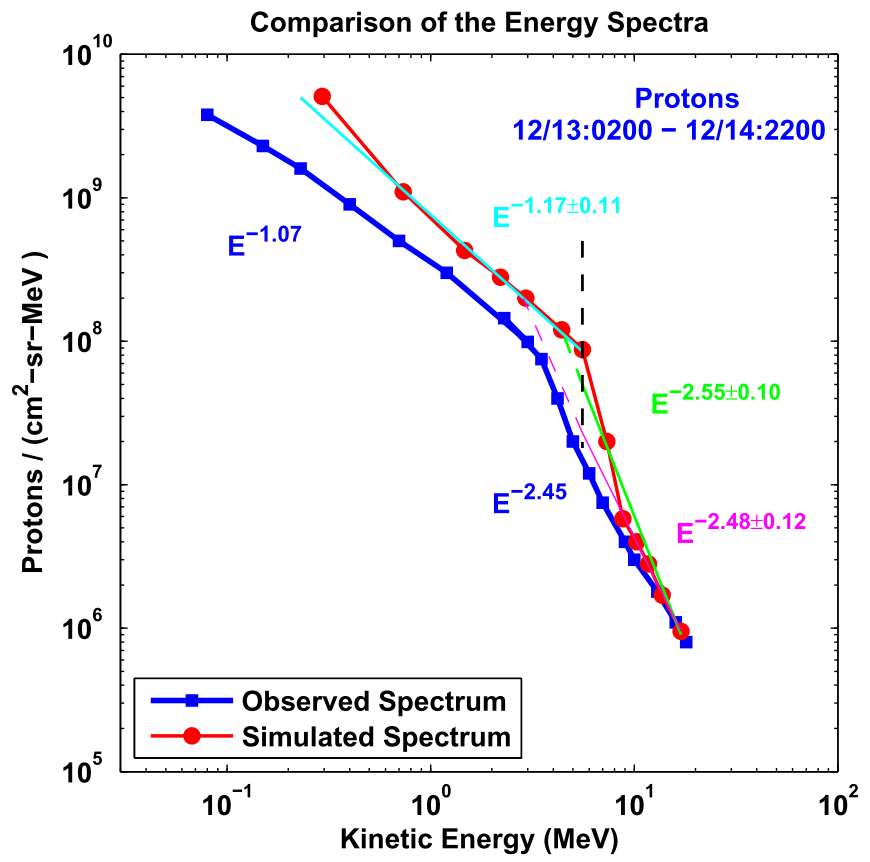

Figure 5. Comparison between the simulated proton energy spectrum and the observed one. The red curve represents the simulated energy spectrum, and the blue curve represents the observed one. In the simulated energy spectrum, the cyan line represents the lower energy spectrum, and the green and pink lines fit the higher energy spectrum with two different indices, respectively. The observed spectrum is analyzed from the data from the ACE, STEREO-A, and STEREO-B spacecraft. The simulated energy spectrum is scaled in the same integrating duration as the observed energy spectrum.

the simulated higher energy spectrum, which possesses a smaller deviation from the "break" point at the vertical dashed line than the pink line. The simulated energy spectrum indicated by the red curve in Figure 5, which is calculated from the downstream regions, shows the energy spectral shape at the energy range from 0.1 to $20 \mathrm{MeV}$. The black vertical dashed line shows the energy spectral "break" at the energy point of $\sim 5.5 \mathrm{MeV}$. The cyan line fits the lower energy spectrum with an index value of $\Gamma_{1}=-1.17 \pm 0.11$. At the higher energy range, the green line fits a suitable energy spectrum with an index value of $\Gamma_{2 a}=-2.55 \pm 0.10$. Its extended dashed line crosses the cyan line over the lower energy range at the point of $\sim 4.5 \mathrm{MeV}$. The pink line fits the energy spectrum with an index value of $\Gamma_{2 b}=-2.48 \pm 0.12$, which would be closer to the observed energy spectral shape over the higher energy range; its extended dashed line crosses the cyan line over the lower energy range at the point of $\sim 3 \mathrm{MeV}$. Although this crossing point at $\sim 3 \mathrm{MeV}$ is close to the observed "break" point at $\sim 3.5 \mathrm{MeV}$, it deviates more toward the actual simulated "break" point at $\sim 5.5 \mathrm{MeV}$. The observed energy spectrum indicated by the blue curve in Figure 5 is analyzed from the proton data from the $A C E$ / EPAM, STEREO/LET, and STEREO/HET spacecraft (Mewaldt et al. 2008). The lower energy spectrum and the higher energy spectrum are "broken" by an energy spectral "break" at the energy point of $\sim 3.5 \mathrm{MeV}$.

Comparing the simulated energy spectrum with the observed energy spectrum, both have spectral "breaks" from 3 to $6 \mathrm{MeV}$. At lower energies, the simulated energy spectral index is $\sim 9 \%$ softer than the observed lower energy spectrum with an index of $\Gamma_{\text {obs } 1}=-1.07$. There also exists a difference in the proton fluxes between the simulated energy spectrum and the observed one. At the higher energy range, the simulated energy spectral index $\Gamma_{2 a}$ is $\sim 4 \%$ and $\Gamma_{2 b}$ is $\sim 1.5 \%$ softer than the observed energy spectrum with an index value of $\Gamma_{\text {obs } 2}=-2.45$, respectively. There is a slight difference in the proton fluxes between the simulated energy spectrum and the observed one over the higher energy range. By comparing the simulated energy spectrum with that observed, the simulated spectrum is "broken" at the energy of $\sim 5.5 \mathrm{MeV}$, and at the higher energy range, the index of $\Gamma_{2 a}$ is more fit for the simulated energy spectrum. In addition, at the lower energy range, the greater difference in the fluxes between the simulated energy spectrum and the observed one would be contributed by the difference in the simulated particle density between the initial time and the end of the simulation. If we exclude the density difference in the simulation, we would decrease the difference in the fluxes over the lower energy range between the simulation and the observation. This means that the actual simulated energy spectral "break" would potentially approximate the observed energy spectral "break" at $\sim 3.5 \mathrm{MeV}$. In general, the present simulated proton fluxes and the energy spectral shape consistently agree with the observed energy spectrum over the energy range from 0.1 to $20 \mathrm{MeV}$.

\section{Summary and Conclusions}

In summary, we simulated the interaction of two converging shocks, and studied the subsequent acceleration of particles. The simulated energy spectrum exhibits a spectral "break" at $\sim 5.5 \mathrm{MeV}$, where the "knee-like" spectral slope changes from a harder to a softer power law. Our simulated spectrum had a maximum energy of a few decades of $\mathrm{MeV}$. With the comparison to the previous single shock model, we make some progress in providing evidence for particle acceleration and energy spectral features in interplanetary shocks. So, why did previous efforts with an isolated shock model fail to predict the energy spectral "break"? There are some difficulties. (i) According to the diffusive shock acceleration theory, the acceleration efficiency is determined by the diffusive coefficient. The highest attainable particle energy is dependent on the diffusive length of particles scaled by the size of the precursor region. If we need to allow a single shock simulation to obtain a large extensive energy spectrum, the size of the precursor region should be expanded to be more than hundreds of times the FEB size using the current Monte Carlo method. It would be more difficult for the simulation code to perform this computation. (ii) In terms of a single shock, the efficiency of the shock kinetic energies translating into the particles is limited. The particles need to travel a long time in their diffusive region to gain energy through multiple crossing cycles. So, the low injection rate would lead to the difficulty in forming an extended energy spectrum quickly. (iii) Furthermore, in the single shock model, the diffusive particles do not only need a long time and a long length of the precursor region to achieve an extended energy spectrum, but this diffusive process would also just form a single power law, not a "broken" power-law energy spectrum.

However, we present some possibilities for forming an extended energy spectrum and producing a "broken" slope in the converging shocks model. First of all, the double-shock interaction would provide more kinetic energy injection into the particle acceleration. The high efficiency injection rate excited by the amplified magnetic turbulence from the region of convergence makes the extended energy spectrum possible. Second, 
the double-shock model provides the accelerated particles opportunities to cross the shock more frequently and gain energy faster. With the compressed precursor region, fewer particles participate in the acceleration process, leading to a steeper slope at high energies, resulting in a spectral "break" at about a few $\mathrm{MeV}$. We prove that the shortening precursor region in the double-shock model has a negative effect on the accelerated particles when the CME shock is extending its energy spectrum. The compressed precursor region leads to a steep spectral slope at the higher energy range. Simultaneously, the amplified magnetic field in the region of convergence would enhance the energy spectrum, extending it to a few tens of MeV. So, there should be a mechanism playing an important role in forming the amplified magnetic field. We suggest that the non-resonant hybrid instabilities would contribute to the amplified magnetic field in the shortening region of convergence (Bell 2013).

The present work is supported by the Xinjiang Natural Science Foundation grant No. 2014211A069. This work is also funded by the CAS Key Laboratory of Solar Activity, National Astronomical Observatories, the Key Laboratory of Modern Astronomy and Astrophysics (Nanjing University), Ministry of Education, China Scholarship Council (CSC), XAO Director Foundation, and NSFC (No.11673056 and 11403087). The authors also thank the support from the Supercomputer Center of University of Arizona. In addition, the authors appreciate Prof. J. R. Jokipii and his group in the University of Arizona, Profs. Hongbo Hu and Hong Lu at IHEP, Prof. Xueshang Feng and his group at NSSC, Profs. W. Gan and Haisheng Ji at PMO, Prof Jun Lin at YNAO, Profs. Quanming Lu and Chenglong Shen at USTC of CAS, and Prof. Liuguan Ding at Nanjing University of Information Science and Technology for their many helpful discussions.

\section{References}

Ackermann, M., Ajello, M., Allafort, A., et al. 2013, Sci, 339, 807

Amenomori, M., Bi, X. J., Chen, D., et al. 2009, ApJ, 692, 61

Bell, A. R. 2013, APh, 43, 56

Bykov, A. M., Gladilin, P. E., Ospipov, S. M., Krassilchtchikov, A. M., \& Vladimirov, A. E. 2013a, MNRAS, 429, 2755

Bykov, A. M., Malkov, M. A., Raymond, J. C., Krassilchtchikov, A. M., \& Vladimirov, A. E. 2013b, SSRv, 178, 599

Bykov, A. M., \& Treumann, R. A. 2011, A\&ARv, 19, 42

Desai, M. I., Mason, G. M., Dayeh, M. A., et al. 2016, ApJ, 828, 106

Ellison, D. C., \& Eichler, D. 1984, ApJ, 286, 691

Ellison, D. C., Möbius, E., \& Paschmann, G. 1990, ApJ, 352, 376

Ellsion, D. C., \& Double, G. P. 2004, APh, 22, 323

Ferrand, G., \& Marcowith, A. 2010, A\&A, 510, 101

Giacalone, J., Burgess, D., Schwartz, S. J., \& Ellison, D. C. 1993, ApJ, 402,550

Guo, F., \& Giacalone, J. 2013, ApJ, 773, 158

Knerr, J. M., Jokipii, J. R., \& Ellison, D. C. 1996, ApJ, 458, 641

Liu, Y., Luhmann, J. G., Müller-Mellin, R., et al. 2008, ApJ, 689, 563

Malkov, M. A., Diamond, P. H., Sagdeev, R. Z., et al. 2013, ApJ, 768, 73

Melrose, D. B., \& Pope, M. H. 1993, PASAu, 10, 222

Mewaldt, R. A., Cohen, C. M. S., Cummings, A. C., et al. 2008, Proc. ICRC (Mexico), 1, 107

Prouza, M., \& Smida, R. 2003, A\&A, 410, 1

Schneider, P. 1993, A\&A, 278, 315

Swordy, S. P., Müller, D., Meyer, P., L’Heureux, J., \& Grunsfeld, J. M. 1990, ApJ, 349, 625

Treumann, R. A. 2009, A\&ARv, 17, 409

Treumann, R. A., \& Jaroschek, C. H. 2008, arXiv:0806.4046v1

Vladimirov, A. E., Bykov, A. M., \& Ellison, D. C. 2008, ApJ, 688, 1084

von Rosenvinge, T. T., Richardson, I. G., Reames, D. V., et al. 2009, SoPh, 256, 443

Wang, X., Wang, N., \& Yan, Y. H. 2013, ApJS, 209, 18

Wang, X., \& Yan, Y. H. 2011, A\&A, 530, A92

Wang, X., \& Yan, Y. H. 2012, RAA, 12, 1535

Wang, X., Yan, Y. H., Ding, M. D., Wang, N., \& Shan, H. 2016, RAA, 16,32

Yang, Z., Liu, Y. D., Richardson, J. D., et al. 2015, ApJ, 809, 28 